\title{
Tumores del estroma gastrointestinal (GIST): factores pronósticos de supervivencia tras citorreducción R0
}

\author{
J. M. Sánchez Hidalgo, F. C. Muñoz Casares, S. Rufián Peña, A. Naranjo Torres, R. Ciria Bru, \\ J. Briceño Delgado y P. López Cillero
}

Servicio de Cirugía General y Aparato Digestivo. Hospital Universitario Reina Sofía. Córdoba

\section{RESUMEN}

Objetivo: analizar los posibles factores pronósticos de supervivencia en tumores estromales gastrointestinales c-kit positivo (GIST), tras citorreducción óptima R0.

Pacientes y método: estudio de 35 pacientes intervenidos en nuestra Unidad desde enero 2002 a febrero 2007, con tumores del estroma gastrointestinal CD117/c-kit positivo en los que se alcanzó citorreducción quirúrgica sin residuo tumoral macroscópico. Una base de datos prospectiva nos proporcionó las distintas variables analizadas, de carácter demográfico, anatómico, clínico, histopatológico e inmunohistoquímico, entre otras. El análisis de la supervivencia actuarial se realizó según el método de Kaplan-Meier y el análisis multivariante mediante el método de regresión múltiple de Cox.

Resultados: la supervivencia global a 5 años fue del 77\%, con una supervivencia media de 52 meses. El riesgo de malignidad según la clasificación de Fletcher y el tamaño tumoral mayor de $10 \mathrm{~cm}$, influyeron significativamente de forma negativa sobre la supervivencia de los pacientes, tras el análisis univariante realizado $(\mathrm{p}<0,05)$. La actividad proliferativa Ki-67 mayor del $50 \%$ fue la única covariable con significación estadística en el análisis multivariante. El 20\% de los tumores recurrieron. Sólo 3 pacientes metastáticos recibieron tratamiento adyuvante con mesilato de imatinib, todos ellos con Ki-67 > 50\% y vivos en la actualidad.

Conclusiones: el índice proliferativo Ki-67 podría representar un excelente marcador pronóstico de supervivencia en aquellos pacientes con tumores del estroma gastrointestinal c-kit positivo. Su confirmación y el punto de corte adecuado deberían ser objeto de futuros estudios prospectivos, así como su posible utilidad para seleccionar pacientes candidatos al tratamiento con mesilato de imatinib.

Palabras clave: GIST. c-kit. Neoplasia mesenquimal.

\begin{abstract}
Objective: to analyze the different factors predictive of survival associated with optimal R0-cytoreduction in c-kit-positive gastrointestinal stromal tumors.

Methods: thirty-five patients were operated on in our Oncological Surgery Department from January 2002 to February 2007 because of CD117/c-kit-positive gastrointestinal stromal tumors, and an optimal surgical cytoreduction was obtained without macroscopical residual disease. Demographic, anatomical, clinical, pathological, and immunohistochemical variables were analyzed from a specific database. Survival and multivariate analyses were developed using Kaplan-Meier and multiple Cox regression models, respectively.

Results: five-year overall survival was $77 \%$ with a mean survival of 52 months. Risk of malignant behaviour according to Fletcher's classification and tumor size higher than $10 \mathrm{~cm}$ had a significantly negative influence on overall survival in the univariate analysis $(\mathrm{p}<0.05)$. Proliferative Ki-67 activity higher than 50\% was the only statistically significant variable in the multivariate analysis. Twenty percent of tumors recurred. Only 3 patients with metastatic disease received adjuvant treatment with imatinib mesylate, all of them with Ki-67 >50\% and currently alive.

Conclusions: the poliferative $\mathrm{Ki}-67$ index could represent an excellent predictive factor for survival in patients with c-kit-positive stromal gastrointestinal tumors. Confirmation and an adequate cut-off level should be the main objectives for future prospective studies, mostly focused on the appropriate selection of optimal candidates to imatinib-mesylate-based treatment.
\end{abstract}

Key words: GIST. c-kit. Mesenchymal tumor.

Sánchez Hidalgo JM, Muñoz Casares FC, Rufián Peña S, Naranjo Torres A, Ciria Bru R, Briceño Delgado J, López Cillero P. Tumores del estroma gastrointestinal (GIST): factores pronósticos de supervivencia tras citorreducción R0. Rev Esp Enferm Dig 2007; 99: 703-708. 


\section{INTRODUCCIÓN}

A partir de 1983 se define con el término GIST (Gastrointestinal Stromal Tumors) un subgrupo de tumores del mesénquima gastrointestinal que no presentaban diferenciación neural ni muscular, y que representaban un $2 \%$ del total de los tumores del tracto digestivo (1).

El descubrimiento del gen c-kit, postuló el origen de los GIST en la célula intersticial de Cajal, al demostrar que comparten con ella la positividad para este gen.

La clasificación en base a factores pronósticos y riesgo de malignidad, así como la aplicación del anticuerpo monoclonal Mesilato de Imatinib (inhibidor de la actividad tirosinaquinasa del gen c-kit), en régimen de neoadyuvancia y/o coadyuvancia, han aumentado el interés hacia estos tumores.

Una línea de investigación en desarrollo se basa en identificar factores pronósticos implicados en la supervivencia de estos tumores. Así, frente a los clásicos factores enunciados por Fletcher (2), como son el tamaño y el índice mitótico, emergen otros como el índice de proliferación celular Ki-67, cuya implicación pronóstica es aún controvertida. Analizar la supervivencia, morbilidad y mortalidad de la serie de tumores GIST intervenidos en nuestra Unidad de Cirugía Oncológica en los que se obtuvo una citorreducción R0, sin residuo tumoral macroscópico, es el objetivo del presente estudio, con especial interés en los posibles factores predictores de supervivencia de nuestra serie.

\section{PACIENTES Y MÉTODOS}

\section{Pacientes}

El estudio incluye 35 pacientes con tumores del estroma gastrointestinal (GIST) intervenidos en la Unidad de Cirugía Oncológica del Hospital Universitario Reina Sofía (Córdoba, España) desde enero de 2002 a febrero de 2007, con seguimiento hasta junio de 2007. Los resultados fueron obtenidos de una base de datos prospectiva.

Los criterios de inclusión fueron: a) tumores con origen histopatológico en el estroma gastrointestinal; b) positividad para el marcador inmunohistoquímico CD117 o c-kit; c) criterios de resección oncológica R0 (no residuo tumoral macroscópico); d) ausencia de otras neoplasias malignas asociadas; y e) capacidad funcional o índice de Karnofsky $>70 \%$.

Las variables incluidas en el estudio fueron las siguientes: edad, sexo, origen gastrointestinal anatómico, presencia o ausencia de síntomas, presencia o ausencia de metástasis, tamaño ( $<2 \mathrm{~cm}, 2-5 \mathrm{~cm}, 5-10 \mathrm{~cm},>10 \mathrm{~cm})$, subtipo histológico (fusocelular, epitelioide o mixto), índice mitótico $\left(\mathrm{n}^{\circ}\right.$ mitosis por cada 50 campos de gran aumento: $\leq 5 \mathrm{~m} / 50$ CGA, 5-10 m/50 CGA, > $10 \mathrm{~m} / 50 \mathrm{CGA})$, potencial riesgo de malignidad, atipia celular (ausente, leve, moderada, intensa), presencia o ausencia de necrosis, hemorragia o ulceración tumoral, estudio inmunohistoquímico (CD34, actina de músculo liso (SMA), vimentina, enolasa (NSE), gluco- proteína s-100, desmina, índice proliferativo Ki-67), estancia postoperatoria, morbilidad y mortalidad quirúrgicas, recurrencia tumoral, tratamiento con imatinib, supervivencia en meses y estado actual.

Las muestras tumorales obtenidas tras su fijación y endurecimiento en formol neutro al $10 \%$, fueron procesadas e incluidas en parafina. Se obtuvieron secciones histológicas que fueron desparafinadas con xilol y posteriormente teñidas con hematoxilina-eosina para su posterior evaluación. Los tumores fueron clasificados según grupos de riesgo, basándose en el potencial riesgo de malignidad propuesto por Fletcher, en relación con las Guías de Consenso del Instituto Nacional de Salud (NIH) (2): riesgo muy bajo, riesgo bajo, riesgo intermedio, riesgo alto y malignos.

\section{Inmunohistoquímica}

Todas las muestras tumorales fueron examinadas frente a distintos anticuerpos inmunohistoquímicos, utilizando preparados comerciales disponibles para ello: CD 117-c-kit (A4502, polyclonal, Dako, EE.UU.; 1:50 dilution), CD34 (clone QBEnd/10. Novocastra Labs; 1:50), SMA (clone asm-1. Dako; 1:200), NSE (clone 5E2: Novocastra Labs; 1:100), S-100 (clone S1/61/69. Novocastra Labs; 1:50), desmina (clone DE-R-11. Novocastra Labs; 1:100).

El índice proliferativo Ki-67 (clon MIB-1, 1:25 dilución) fue determinado con el procedimiento estándar: se estimó el número de células MIB-1 determinado por contaje de 1.000 células en las áreas de mayor actividad y con una magnificación x 400. Se procedió a clasificar según los diferentes estratos en: baja actividad proliferativa, cuando el porcentaje es inferior al 10\%; moderada actividad proliferativa, cuando el porcentaje es entre el 10 y el $50 \%$ y alta actividad proliferativa, cuando el porcentaje es superior al $50 \%$.

\section{Análisis estadístico}

Para los cálculos estadísticos se utilizó el programa informático SPSS 11.0 para Windows (Microsoft ${ }^{\circledR}$, EE.UU.). Las diferencias entre frecuencias y medias se obtuvieron mediante los métodos de Chi cuadrado, t de Student y análisis de la varianza. El análisis de la supervivencia se llevó a cabo según el método de Kaplan-Meier y el análisis multivariante mediante el método de regresión múltiple de Cox. En todas las pruebas estadísticas se consideraron valores "significativos" aquellos cuyo nivel de confianza fue del $95 \%$, es decir, un error alfa inferior a $0,05(\mathrm{p}<0,05)$.

\section{RESULTADOS}

\section{Resultados descriptivos}

Durante el periodo en que se llevó a cabo el estudio, se diagnosticaron 1.720 tumores digestivos en el Hospital 
Reina Sofía, de los cuales 38 casos eran tumores del estroma gastrointestinal (GIST). Treinta y cinco pacientes reunían los criterios de selección y fueron incluidos en el estudio. Tres pacientes fueron excluidos, dos de ellos por presentar otras neoplasias malignas concomitantes, que impedían un seguimiento adecuado y otro paciente por presentar un índice de Karnofsky $<70 \%$. La edad media de presentación fue 60 años (rango: 25-84), siendo más frecuente la presentación entre la $6^{\mathrm{a}}$ y $7^{\mathrm{a}}$ décadas. El $54 \%$ fueron mujeres, frente al $46 \%$ de hombres. La localización anatómica preferente fue gástrica $(48 \%)$ e intestino delgado $(46 \%)$, seguida en menor frecuencia del mesenterio (6\%). El $86 \%$ de los pacientes fueron sintomáticos, predominando entre los síntomas referidos la hemorragia digestiva alta, síndrome constitucional, anemia y dolor abdominal. El 23\% presentaron metástasis a distancia en el diagnóstico, la mitad en hígado y la otra mitad en peritoneo (Tabla I).

El tamaño medio fue $9,4 \mathrm{~cm}$, con una mediana de $8 \mathrm{~cm}$ (rango: 2-33). El 60\% de los pacientes superaron los $5 \mathrm{~cm}$, y el $29 \%$ los $10 \mathrm{~cm}$. En cuanto a la estirpe celular fue mixta (epitelioide-fusocelular) en casi la mitad de los pacientes, epitelioide en un $29 \%$ y fusocelular en el $23 \%$.
La tumoración presentaba signos de hemorragia en un $37 \%$, necrosis en un 34\%, y ulceración en $11 \%$.

El índice mitótico fue inferior a 5 mitosis/50 CGA en el $66 \%$ de los pacientes, comprendido entre 6-10 mitosis/50 CGA en un $11 \%$, y mayor de 10 mitosis/50 CGA en el $23 \%$ restante. En el $43 \%$ de los casos existía algún grado de atipia celular, siendo de carácter intenso en el $20 \%$. La clasificación de los tumores extirpados en relación al riesgo de malignidad, mostró los siguientes valores: riesgo muy bajo $0 \%$, riesgo bajo $34 \%$, riesgo intermedio $23 \%$, riesgo elevado $20 \%$ y maligno $23 \%$.

El estudio inmunohistoquímico demostró positividad para CD117 en el 100\% de los pacientes, como criterio de inclusión que era, siendo CD34 el siguiente en orden descendente de frecuencia (69\%), seguido de actina del músculo liso $(46 \%)$, vimentina $(6 \%)$, enolasa $(6 \%)$, prot s-100 (3\%) y desmina (3\%). En cuanto a la actividad proliferativa $\mathrm{Ki}-67:>10 \%$ en el $46 \%$ de pacientes, $10-50 \%$ en $31 \%$ de pacientes, $y>50 \%$ en el $23 \%$ restante.

La estancia hospitalaria mediana postoperatoria fue de 8 días, con un rango comprendido entre 4 y 26 días. La morbilidad postoperatoria fue del $14 \%$ debido a la presencia postquirúrgica de dos abscesos intraabdominales, una

Tabla I. Pacientes intervenidos con tumor del estroma gastrointestinal c-kit positivo (GIST) y metástasis

\begin{tabular}{|c|c|c|c|c|c|c|c|c|c|c|}
\hline$N^{\circ}$ & Sexo & $\begin{array}{l}\text { Edad } \\
\text { (años) }\end{array}$ & Localización & $\begin{array}{l}\text { Tamaño } \\
(\mathrm{cm})\end{array}$ & $\begin{array}{l}\text { Mitosis } \\
\text { (I5OHPF) }\end{array}$ & $\begin{array}{l}\text { Ki-67 } \\
(\%)\end{array}$ & $\begin{array}{l}\text { Lugar } \\
\text { metástasis }\end{array}$ & $\begin{array}{l}\text { Cirugía } \\
\text { realizada }\end{array}$ & $\begin{array}{c}\text { Tratamiento } \\
\text { imatinib }\end{array}$ & $\begin{array}{l}\text { Supervivencia } \\
\text { (meses) }\end{array}$ \\
\hline 1 & M & 65 & Estómago & $>10$ & $>10 / 50$ & $>50$ & Peritoneo & $\begin{array}{c}\text { Gastrectomía } \\
+ \\
\text { peritonectomía }\end{array}$ & No & Muerto (37) \\
\hline 6 & M & 68 & Intestino delgado & $>10$ & $>10 / 50$ & $5-10$ & Hígado & $\begin{array}{c}\text { Yeyunectomía } \\
+ \\
\text { hepatectomía derecha }\end{array}$ & No & Vivo (61) \\
\hline 10 & $\mathrm{~F}$ & 74 & Intestino delgado & $2-5$ & $6-10$ & $>50$ & Peritoneo & $\begin{array}{c}\text { Yeyunectomía } \\
+ \\
\text { peritonectomía }\end{array}$ & No & Vivo (47) \\
\hline 21 & M & 52 & Estómago & $5-10$ & $>10 / 50$ & $>50$ & Hígado & $\begin{array}{c}\text { Gastrectomía } \\
+ \\
\text { metastasectomía }\end{array}$ & No & Muerto (15) \\
\hline 25 & $\mathrm{~F}$ & 37 & Intestino delgado & $5-10$ & $>10 / 50$ & $>50$ & Hígado & $\begin{array}{c}\text { Yeyunectomía } \\
+ \\
\text { hepatectomía derecha }\end{array}$ & $\begin{array}{c}\text { Sí } \\
\text { (adyuvante) }\end{array}$ & Vivo (23) \\
\hline 27 & $\mathrm{~F}$ & 67 & Estómago & $>10$ & $>10 / 50$ & $>50$ & $\begin{array}{c}\text { Peritoneo } \\
\text { Bazo }\end{array}$ & $\begin{array}{c}\text { Gastrectomía } \\
+ \\
\text { esplenectomía }\end{array}$ & No & Muerto (7) \\
\hline 30 & $\mathrm{~F}$ & 27 & Intestino delgado & $>10$ & $>10 / 50$ & $>50$ & Peritoneo & $\begin{array}{c}\text { Yeyunectomía } \\
+ \\
\text { peritonectomía }\end{array}$ & $\begin{array}{c}\text { Sí } \\
\text { (adyuvante) }\end{array}$ & Vivo (14) \\
\hline 33 & $\mathrm{~F}$ & 41 & Estómago & $>10$ & $>10 / 50$ & $>50$ & Hígado & $\begin{array}{c}\text { Gastrectomía + } \\
+ \\
\text { segmentectomía hepática }\end{array}$ & $\begin{array}{c}\text { Sí } \\
\text { (adyuvante) }\end{array}$ & Vivo (9) \\
\hline
\end{tabular}


atelectasia, una neumonía y una infección de herida quirúrgica. No hubo ninguna mortalidad quirúrgica en esta serie de pacientes analizada. Sólo 3 pacientes $(9 \%)$ han recibido tratamiento con mesilato de imatinib, todos ellos pacientes con actividad proliferativa Ki-67 $>50 \%$ y considerados malignos por ser metastásicos. En los tres pacientes se administró en régimen de adyuvancia, estando actualmente vivos y sin recurrencia. Siete pacientes (20\%) han presentado recurrencia del tumor, localmente o a distancia, siendo todos ellos tumores clasificados en riesgo alto de malignidad o malignos, según la clasificación de Fletcher.

\section{Resultados de supervivencia}

La supervivencia global a 3 y 5 años, estimada por el método de Kaplan Meier, fue del 83 y $77 \%$ respectivamente, con una supervivencia media de 52 meses. No hubo diferencias significativas de supervivencia a los 5 años en referencia al sexo ( $89 \%$ en mujeres $v s .70 \%$ en hombres, $\mathrm{p}=0,50)$, ni al origen anatómico gástrico $v s$. intestino delgado (67 vs. 86\% respectivamente, $\mathrm{p}=0,34$ ). En este mismo sentido, la presencia o no de síntomas en el momento del diagnóstico tampoco mostró diferencias en la supervivencia a 5 años $(77 v s .75 \%, \mathrm{p}=0,88)$.

En cuanto a la existencia de metástasis sincrónicas, aunque resecables, influyeron en la supervivencia a 5 años, frente a aquellos pacientes que no las presentaban, aunque sin completa significación estadística (47 vs. 87\%, $\mathrm{p}=0,05)$. Algo similar ocurrió al analizar la supervivencia en relación con el índice mitótico, estableciendo el punto de corte en 10 mitosis por cada 50 campos de gran aumento, siendo del $87 \%$ a 5 años en el grupo de $\leq 10 / 50$ CGA con una supervivencia media de 53 meses, frente al $47 \%$ en el grupo con $>10 / 50$ CGA y una supervivencia media de 41 meses (Fig. 1).

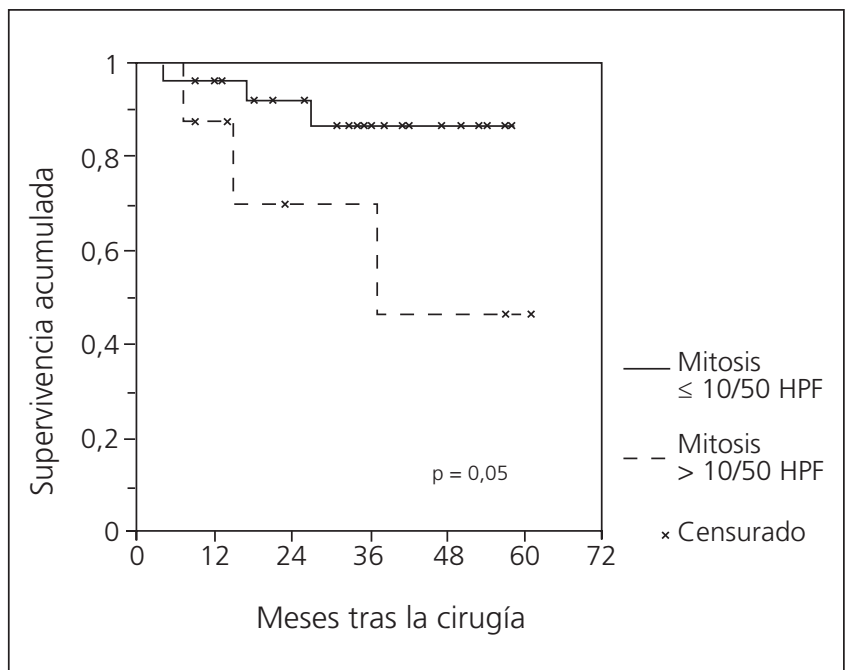

Fig. 1. Análisis de supervivencia según método de Kaplan-Meier de los pacientes intervenidos de tumor GIST c-kit positivo, en relación al índice mitótico.
La recurrencia del tumor estromal GIST durante el seguimiento, localmente o a distancia, se asoció con peor supervivencia, pero sin significación estadística. Así, aquellos pacientes con recurrencia tumoral evidente presentaron una supervivencia a 5 años del $33 \%$, frente al $88 \%$ de los que no recurrieron $(\mathrm{p}=0,06)$. Estos pacientes han sido reintervenidos en su mayoría (4/7).

En cambio, sí mostraron diferencias significativas de supervivencia la asociación de los grupos de riesgo muy bajo, bajo e intermedio, según Fletcher, frente a la asociación de los grupos de riesgo alto y malignos (95 vs. $54 \%$, respectivamente) (Fig. 2). También el tamaño tumoral fue otra de las variables que influyó en la supervivencia a 5 años. Estableciendo el punto de corte en $10 \mathrm{~cm}$, encontramos que la supervivencia de aquellos tumores $\leq 10 \mathrm{~cm}$ era superior a la alcanzada cuando el tumor medía $>10 \mathrm{~cm}$ (91 vs. $31 \%$ respectivamente, $\mathrm{p}=0,01)$, con una supervivencia media de 54 meses frente a 37 meses (Fig. 3).

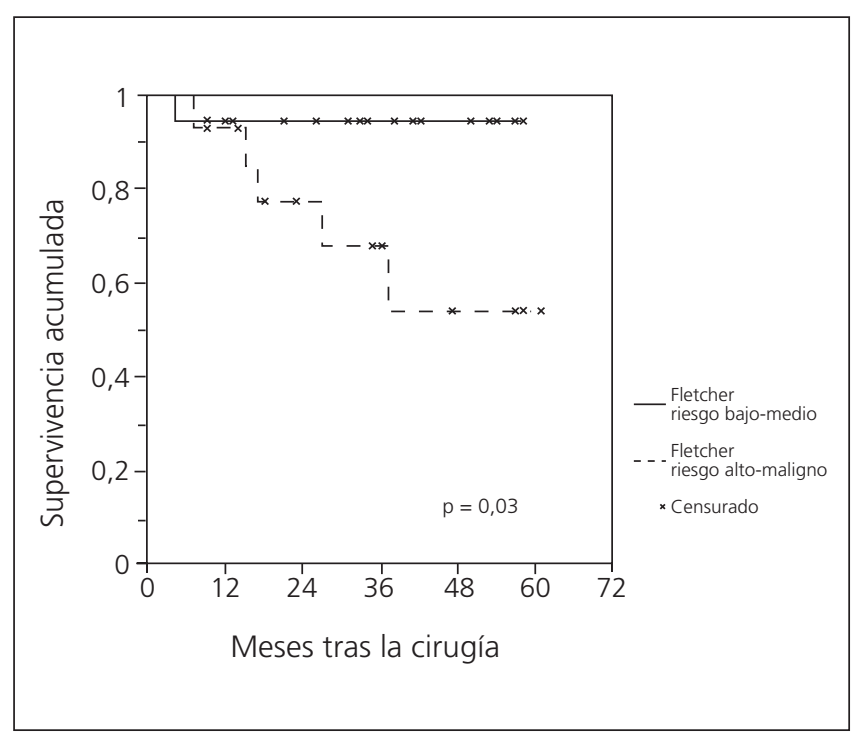

Fig. 2. Análisis de supervivencia según método Kaplan-Meier de los pacientes intervenidos de tumor GIST c-kit positivo, en relación al riesgo de malignidad según clasificación de Fletcher.

El análisis de supervivencia, según los distintos marcadores de inmunohistoquímica, no mostró diferencias estadísticamente significativas, salvo en relación a CD34 y actina del músculo liso. En cambio, sí mostró diferencias al analizar Ki-67, cuando el punto de corte analizado era la actividad proliferativa Ki-67 $\leq 50 \%$ frente a Ki-67 $>50 \%$ (92 vs. 23\%, respectivamente, p =0,001) (Fig. 4). Esta covariable fue la única que mostró significación estadística tras el análisis multivariante realizado mediante el método de regresión múltiple de Cox $(\mathrm{B}=1.976$; Wald $=3,718 ; \mathrm{p}=0,045$; Odss ratio $=7,214 ;$ IC95\% $=0,968$ $53,765)$. En este sentido, la actividad proliferativa Ki-67 $\leq 50 \%$ se ha mostrado como la única covariable predicto- 


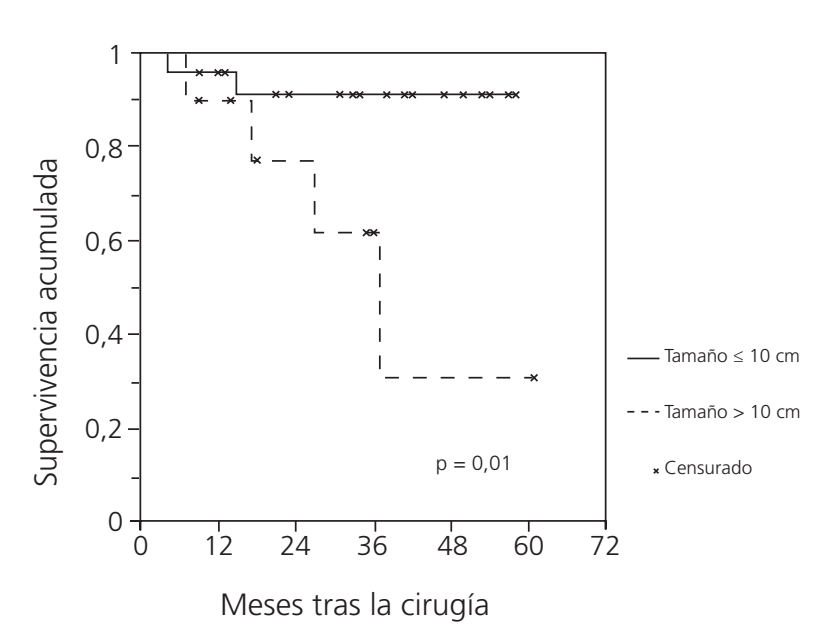

Fig. 3. Análisis de supervivencia según método de Kaplan-Meier de los pacientes intervenidos de tumor GIST c-kit positivo, en relación al tamaño tumoral menor o igual a $10 \mathrm{~cm}$ frente a mayor a $10 \mathrm{~cm}$.

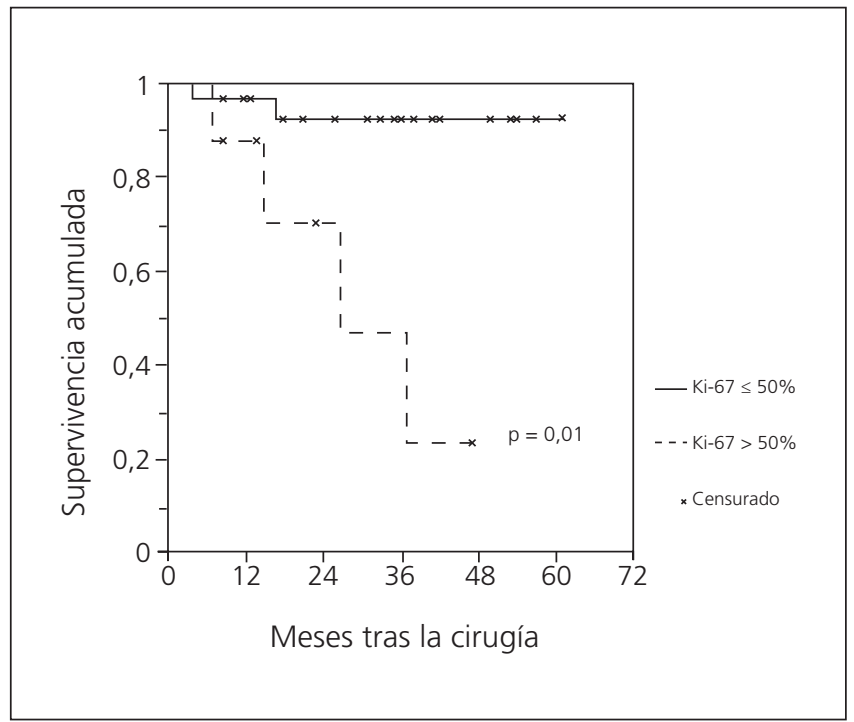

Fig. 4. Análisis de supervivencia según método de Kaplan-Meier de los pacientes intervenidos de tumor GIST c-kit positivo, en relación al porcentaje de actividad proliferativo Ki-67.

ra de supervivencia en nuestra serie de tumores estromales gastrointestinales (GIST) sometidos a cirugía citorreductora R0.

\section{DISCUSIÓN}

Uno de los inconvenientes para estudiar los tumores del estroma gastrointestinal es su baja incidencia. La positividad para CD117, en menor medida para CD34, así como el origen del tumor en el tracto gastrointestinal, permiten afirmar con certeza que estamos ante un GIST y no ante un leiomioma o leiomiosarcoma (3).
En nuestra serie, los resultados encontrados con respecto a edad y sexo concuerdan con la literatura, reflejando una incidencia poco frecuente antes de los 40 años (4), así como igualdad entre ambos sexos (5). En cuanto a la forma de presentación clínica, tampoco se mostraron grandes diferencias con respecto a otras series publicadas, predominando los pacientes sintomáticos con hemorragia digestiva, dolor abdominal y sensación de masa abdominal frente a los casos incidentales $(6,7)$. Por otra parte, es conocido que los GIST tienen similar predilección por originarse en estómago e intestino delgado (8) y aunque existen estudios que describen un mejor pronóstico para tumores de localización gástrica, a igualdad de tamaño y número de mitosis (9), nosotros no evidenciamos diferencias significativas entre los distintos grupos. Al contrario que otros estudios $(5,9)$, en nuestra serie predominó el subtipo histológico mixto epitelioide-fusocelular, aunque sin repercusión en la supervivencia.

En base a nuestros resultados podemos confirmar al hígado y, en segundo lugar, al peritoneo, como las localizaciones de predominio para las metástasis (10). En la misma línea que otros autores, no evidenciamos que la necrosis, ulceración y hemorragia tumoral tengan relación con un mayor riesgo de malignidad $(9,11) \mathrm{y}$, compartimos con Miettinen, que la atipia nuclear no muestra relación con la supervivencia y el riesgo de malignidad (9).

En cuanto a la positividad para los diferentes marcadores inmunohistoquímicos, existe concordancia con los porcentajes publicados por otras series, situando a CD34 y actina del músculo liso, tras CD 117, como los más representativos para los GIST $(12,13)$.

La supervivencia media a los 5 años, superando el $75 \%$, para GIST con resección oncológica completa (R0), se manifiesta en consonancia con los mejores resultados publicados por otros autores (14-16). Con respecto a la tasa de recurrencias y mediana de supervivencia de los tumores R0 recidivados, nuestra serie muestra unos resultados sensiblemente mejores al $40 \%$ y 2 años, respectivamente, destacados por otros grupos (17). La supervivencia mediana de 37 meses en nuestros casos es explicable por tratarse de recurrencias potencialmente resecables en 5 de los 7 casos descritos por nuestro estudio. Esta segunda resección permite aumentar en 12-18 meses la supervivencia media, tal como describía Bonvalot (18).

La importancia del índice mitótico como marcador pronóstico es ampliamente reconocido, especialmente cuando es superior a 10 mitosis por cada 50 CGA $(19,20)$. Los resultados en nuestra serie no fueron estadísticamente significativos al relacionarlo con la supervivencia $(p=0,05)$, aunque creemos que un aumento en el tamaño muestral sería suficiente para que el test alcanzase la significación estadística.

El tamaño es muy variable en nuestra muestra, con valores entre 1 y $30 \mathrm{~cm}$ con una mediana de $8 \mathrm{~cm}$. Establecer el punto de corte para análisis de supervivencia en $10 \mathrm{~cm}$ marca diferencias en el pronóstico de estos tumo- 
res: $90 v s .30 \%$ a los 5 años en nuestra serie y en otras series como la de Orosz (11).

En función del índice de Fletcher, hemos observado que el pronóstico de tumores de bajo potencial maligno es excelente. Sin embargo, en tumores con alto potencial maligno o malignos inicialmente, la tasa de supervivencia es baja oscilando entre el 20 y el $50 \%(7,20)$. A pesar de ello, no es posible con certeza predecir a largo plazo el comportamiento de GIST de bajo riesgo, pues un $20 \%$ de tumores con tamaño inferior a $5 \mathrm{~cm}$ e índice mitótico $<5 / 50$ CGA, metastatizan $(2,4)$.

El índice proliferativo Ki-67, de reciente uso en GIST, es referido como indicador de mal pronóstico, al igual que el índice mitótico, aunque con una relevancia controvertida, según diferentes estudios (21-24). Probablemente sea esta una de las mayores aportaciones que permite nuestro estudio. En este sentido, un índice proliferativo Ki-67 > 50\% se mostró como factor de mal pronóstico al relacionarlo con la supervivencia global tanto en el análisis univariante $(\mathrm{p}=0,001)$ como multivariante $(\mathrm{p}<0,1)$. Estos datos coinciden con estudios como el de Özguc (3), donde se establece un índice proliferativo Ki-67 > 82\% como marcador pronóstico de supervivencia. Sin embargo, serán necesarios nuevos estudios prospectivos que permitan establecer en un futuro, el adecuado porcentaje del índice proliferativo Ki-67, muy variable en los diferentes trabajos (10).

En resumen, en la actualidad podemos decir que la cirugía es la única posibilidad potencialmente curativa en GIST. Obtener márgenes quirúrgicos libres R0 debe primar sobre las exéresis funcionales. Así mismo, las recidivas potencialmente resecables deben ser extirpadas al demostrar una menor tasa de recurrencias y un aumento en la supervivencia. Las aplicaciones de imatinib mesilato deben ir ligadas siempre a la resecabilidad del tumor (25), ya sea en régimen neoadyuvante, adyuvante o paliativo, dirección en la que se centran los ensayos clínicos multicéntricos puestos en marcha actualmente. La inclusión en estudios prospectivos y multicéntricos de Ki-67, estudiando su relación con índices de riesgo como el de Fletcher, podría definir con mayor precisión el riesgo de malignidad y recurrencia de estos tumores, así como establecer candidatos a terapias adyuvantes o neoadyuvantes con mesilato de imatinib.

\section{BIBLIOGRAFÍA}

1. Mazur MT, Clark HB. Gastric stromal tumors. Reappraisal of histogenesis. Am J Surg Pathol 1983; 7: 507-19.

2. Fletcher CD, Berman JJ, Corless C, Gorstein F, Lasota J, Longley BJ, et al. Diagnosis of gastrointestinal stromal tumours: A consensus approach. Hum Pathol 2002; 33: 459-65.

3. Özguç H, Yilmazlar T, Yerci Ö, et al. Analysis of prognostic and in- munohistochemical factors in gastrointestinal stromal tumors with malignant potential. J Gastrointest Surg 2005; 9 (3): 418-29.

4. Demetri GD. Targeting c-kit mutations in solid tumours: Scientific rationale and novel therapeutic options. Sem Oncol 2001; (Supl. 17): $19-26$.

5. Miettinen M, Majidi M, Lasota J. Pathology and diagnostic criteria of gastrointestinal stromal tumours (GISTs): A review. Eur J Cancer 2002; 38: S39-S51.

6. De Francisco R, Díaz G, Cadahía V, Velásquez RF, Giganto F, González O, et al. Lower GI bleeding secondary to a stromal rectal tumor (rectal GIST). Rev Esp Enferm Dig 2006; 98: 387-8.

7. Fernandez Salazar T, Álvarez Gago A, Sanz Rubiales B, Velayos Jiménez R, Aller de la Fuente, González Hernández JM. Gastrointestinal stromal tumors (GISTs): Clinical aspects. Rev Esp Enferm Dig 2007; 99: 19-24

8. Nilsson B, Bümming P, Meis-Kindblom JM, Odén A, Dortok A, Gustavsson B, et al. Gastrointestinal stromal tumors: The incidence, prevalence, clinical course, and prognostication in the preimatinib mesylate era. A population-based study in western Sweden. Cancer 2005; 103 (4): 821-9.

9. Miettinen M, El-Rifai W, Sobin LH, et al. Evaluation of malignancy and prognosis og gastrointestinal stromal tumours: A review. Hum Pathol 2002; 33: 478-83.

10. Roberts PJ, Eisenberg B. Clinical presentation of gastrointestinal stromal tumors and treatment of operable disease. Eur J Cancer 2002; 38 (Supl. 5): S37-8.

11. Orosz Z, Tornoczky T, Sapi Z. Gastrointestinal stromal tumors: A Clinicopathologic and Inmunohistochemical Study of 136 Cases. Pathology Oncology Research 2005; 11: 11-21.

12. Tworek JA, Appelman HD, Singleton TP, Greenson JK. Stromal tumors of the jejunum and ileum. Mod Pathol 1997; 10 (3): 200-9.

13. D'Amato G, Steinert DM, McAuliffe JC, Trent JC. Update on the Biology and Therapy of Gastrointestinal Stromal Tumors. Cancer Control 2005; 12 (1): 44-56.

14. Miettinen M, Sobin LH, Sarlomo-Rikala M. Inmunohistochemical spectrum of GISTs at different sites and their differential diagnosis with a reference to CD117 (KIT). Mod Pathol 2000; 13: 1134-42.

15. Nagasaki Y, Misawa K, Kohashi S, et al. Evaluation of malignantancy using Ki-67 prognostic index for gastric stromal tumor. Gastric Cancer 2003; 6: 168-72.

16. Kim CJ, Day S, Yeh KA. Gastrointestinal stromal tumors: Análisis of clinical and pathological factors. Am Surg 2001; 67: 135-7.

17. DeMatteo RP, Lewis JJ, Leung D, et al. Two hundred gastrointestinal stromal tumors. Recurrence patterns and prognostic factors for survival. Ann Surg 2000; 232: 51-8.

18. Bonvalot S. Surgical management of GIST in the era of Gleevec. Annales de Chirurgie 2005; 130 (3): 144-51.

19. Evans HL. Smooth muscle tumors of the gastrointestinal tract: A study of 56 cases followed for a minimum of 10 years. Cancer 1985; 56: $2242-9$

20. Emory TS, O`Leary TJ. Prognosis and surveillance of gastrointestinal stromal/smooth muscle tumours. Ann Chir Gynaecol 1998; 87: 30610.

21. Suster S. Gastrointestinal stromal tumors. Semin Diagn Pathol 1996; 13: 297-313.

22. Panizo-Santos A, Sola I, Vega F, et al. Predicting metastastasic risk of gastroinetstinal stromal tumors: role of cell proliferation and cell cycle regulatory proteins. Int J Surg Pathol 2000; 8: 133-44.

23. Rudolph P, Gloeckner K, Parwaresch R, et al. Inmunophenotype, proliferation, DNA ploidy, and biological behavior of gastrointestinal stromal tumors: A multivariate clinico-pathologic study. Hum Pathol 1998; 29: 791-800.

24. Goldblum JR, Appleman HD. Stromal tumors of the duodenum. A histologic and inmunohistochemical study of 20 cases. Am J Surg Pathol 1995; 19: 71-80.

25. Dematteo RP, Heinrich MC, El-Rifai WM, Dimetri G. Clinical management of gastrointestinal stromal tumours: Before and after of STI571. Hum Pathol 2002; 33: 466-77. 